\title{
Claude Simon et André Vick-Mengus : matière photographique et affinités primitivistes
}

Claude Simon and André Vick-Mengus: Photographic Subject Matter and Primitivist Affinities

\section{Laura Laborie}

\section{(2) OpenEdition}

\section{Journals}

Édition électronique

URL : https://journals.openedition.org/ccs/2958

DOI : $10.4000 /$ ccs. 2958

ISSN : 2558-782X

Éditeur :

Presses universitaires de Rennes, Association des lecteurs de Claude Simon

Édition imprimée

Date de publication : 3 septembre 2020

Pagination : $155-170$

ISBN : 978-2-7535-8065-7

ISSN : $1774-9425$

\section{Référence électronique}

Laura Laborie, «Claude Simon et André Vick-Mengus : matière photographique et affinités

primitivistes », Cahiers Claude Simon [En ligne], 15 | 2020, mis en ligne le 03 septembre 2021, consulté le 05 décembre 2022. URL : http://journals.openedition.org/ccs/2958 ; DOI : https://doi.org/10.4000/ ccs. 2958 


\title{
CLAUDE SIMON ET ANDRÉ VICK-MENGUS : MATIĖRE PHOTOGRAPHIQUE ET AFFINITÉS PRIMITIVISTES
}

\author{
Laura LABORIE
}

En 1957, Claude Simon publie Le Vent empruntant certains éléments au réel pour bâtir l'intrigue de son roman. Suite à un héritage, un étranger devient exploitant agricole dans une ville du Midi et suscite par sa seule manière d'être l'indignation des habitants, engendrant malgré lui une série de péripéties malheureuses. Pour créer cette figure singulière qu'est Antoine Montès, Claude Simon s'est inspiré de son cousin par alliance : André Vick-Mengus. L'aspect physique est révélateur sur ce point; ce sont les cheveux " trop longs de dix centimètres " et " dépassant du béret "; sont aussi évoqués le vélo qu'il enfourche, sa « dégaine à la fois simiesque et aristocratique ( $V$, resp. p. 7, 164 et 45), et, bien sûr, l'appareil photographique accroché à son cou. En effet, Mengus déclare dans une lettre adressée à Simon : "Tout est tiré de moi : aspect extérieur, propos, et même [l]es aventures [...]. Tout a en moi sa racine. Tout concourt à un double portrait, extérieur et intérieur, à la fois minutieux et déformé ${ }^{1}$. "

Lors de la parution du Vent, Mengus reproche à Simon de s'être inspiré de sa vie personnelle sans avoir pris soin de demander son approbation. Suivra une brouille passagère qui sépare les deux hommes : Mengus insiste pour que le roman soit retiré de la vente afin d'apporter des corrections masquant les aspects qu'il juge calqués sur le réel tandis que Simon refuse en bloc toute modification, ne percevant pas dans quelle mesure ce qu'il a voulu comme un portrait laudatif, un hommage à quelqu'un qu'il estime profondément, puisse sembler

1. Lettre d'André Mengus à Claude Simon, Salses, 3 octobre 1957, archives aimablement mises à disposition par Florence Lacour-Bourgoin. 
blessant ou ironique. L'écrivain précise à son sujet : « [c’est] un des rares êtres humains avec lesquels je sente une réelle affinité et pour lesquels j'éprouve une vraie affection ${ }^{2}$ ». Face à de telles déclarations, on ne peut que se montrer curieux d'en savoir un peu plus sur l'individu charismatique qu'était André Mengus afin de découvrir les affinités qui liaient les deux cousins. Quelques éléments biographiques nous permettront de dresser un portrait plus précis.

Issu d'une famille bourgeoise alsacienne, André Mengus est né à Strasbourg en 1904; lorsque sa mère divorce, il part vivre à Paris chez ses grands-parents maternels. En 1912, sa mère épouse l'écrivain, peintre et poète Louis Codet qui n'est autre que le cousin de Suzanne Denamiel, la mère de Claude Simon. L'admiration de l'enfant pour son beau-père sera telle que, bien des années plus tard, il envisage de transformer l'hôtel particulier situé rue de la Cloche d'or en musée dédié à la mémoire de Louis Codet. Durant sa jeunesse, il entreprend des études d'art à l'école du Louvre, mais, suite à différents accidents qui l'affectent physiquement, il doit renoncer à ses déplacements à l'étranger et s'accommoder d'une santé fragile. Pendant la guerre, il part vivre dans le Perche, à Bellême, et commence à réaliser, en autodidacte, des photographies à l'aide d'un Rolleiflex. Au même moment, il hérite de Louis Codet une importante propriété viticole à Salses; c'est donc dans les années quarante que Mengus découvre pour la première fois le Roussillon dont il n'avait entendu parler jusqu'alors que par les récits de son beau-père. L'attachement à ce territoire est immédiat, il décide de s'installer dans la maison familiale de Salses et devient exploitant agricole, tout en poursuivant ses travaux photographiques.

En 1940, Claude Simon s'évade du camp de prisonniers allemand pour se réfugier rue de la Cloche d'or à Perpignan. Vivant à proximité, les deux hommes, âgés respectivement d'une trentaine et d'une quarantaine d'années, se fréquentent assidûment pendant plus de quinze ans, partageant une passion commune pour la photographie. Simon a découvert ce médium artistique ${ }^{3}$ avec la revue surréaliste Minotaure, publiant les "Danseuses » dans la revue

2. Lettre de Claude Simon à André Mengus, Paris, 26 septembre 1957, archives mises à disposition par F. Lacour-Bourgoin.

3. Sur cette question, voir notamment I. Albers, Moments photographiques, Villeneuve d'Ascq, Presses universitaires du Septentrion, 2007; M. Calle-Gruber, Le Grand temps. Essai sur Claude Simon, Villeneuve d'Ascq, Presses universitaires du Septentrion, 2004; J. H. Duffy (qui est la première à consacrer une étude à Photographies), "Claude Simon's Photographies : A Formal and Thematic Analysis ", Nottingham French Studies, 33/2, 1994, et sa traduction française, "Analyse formelle et thématique de Photographies de Claude Simon ", Les Images chez Claude Simon. Des mots pour le voir, La Licorne, n 71, PUR, 2004, p. 133-151. 
Verve en 1938. Il enrichit et développe son univers esthétique au contact de ce cousin fantasque, qui lui fournit de nouveaux motifs à explorer. Ils cheminent alors côte à côte dans les rues de la ville catalane et dans les alentours.

Esthète raffiné, André Mengus possède des caractéristiques propres aux auteurs d'Art Brut. Son travail s'est construit loin de toute école et il n'a pas cherché à se faire connaître. Se moquant de la notoriété, il ne publie qu'un seul ouvrage, Les Petits des Hommes, en 1954, qu'il signe de son nom d'artiste "André Vick ». Parce qu'il publie dans des revues catalanes et organise de nombreuses expositions à Perpignan, Mengus ne diffusant pas son travail en dehors du Roussillon, son œuvre photographique n'est connue que localement. Dans ses carnets, il confesse :

Il me plaît que des photographies ne soient pas, dans un livre, emprisonnées parmi ses blancs et sa typographie, mais intégrées dans une longue phrase, cette phrase en images ne les empêchant pas, au contraire, d'aller au-devant de nos yeux, nous ouvrant un espace en nous donnant l'illusion d'y vivre ${ }^{4}$.

Le rejet de la forme livresque pour l'érudit qu'est Mengus ne doit pas nous tromper. Il s'agit d'une coquetterie d'artiste obéissant à quelque élan primitiviste. Par souci de spontanéité et recherche d'immédiateté, le photographe exige un espace ouvert, non cloisonné où la libre circulation des photographies soit manifeste, permettant à chacun de s'y projeter plus aisément, en dehors de la forme fixe et conventionnelle qu'est le livre.

Lucien Dällenbach est le premier critique à porter un intérêt à ce qu'il nomme « la primordialité ${ }^{5}$ » chez Claude Simon. Ainsi, la présence de l'élémentaire, de l'archaïque, du primitif, innerve son œuvre qui s'inscrit dans le mouvement primitiviste touchant tous les arts au début $\mathrm{du} \mathrm{xx}^{\mathrm{e}}$ siècle. S'intéresser à ce courant esthétique à travers la photographie peut paraître paradoxal tant ce médium artistique marque l'apogée de l'ère industrielle et échappe à toute élémentarité dans sa mise en œuvre technique. Néanmoins, le primitivisme n'est pas le primitif et, comme le rappelle Henri Meschonnic, "le primitivisme est recherche du primitif ${ }^{6}$ ". Avant de s'intéresser à ce mouvement artistique, présenté par les historiens de l'art comme le résultat d'une confrontation entre artistes occidentaux et monde tribal, il convient de définir plus précisément le terme de " primitif », qui signifie " le plus ancien,

4. Les Carnets d'André Vick en Roussillon, " troisième cahier ", Éditions du Castillet, Perpignan, 1968, p. 28.

5. L. Dällenbach, «La question primordiale », dans Sur Claude Simon, Minuit, 1987, p. 68.

6. H. Meschonnic, Modernité, Modernité, Lagrasse, Verdier, 1988, p. 278. 
originel $^{7}$ ", mais qui est « entré dans l'usage courant avec un sens plus ambigu qu'il n’est normal $^{8}$ ». Son équivocité repose sur sa récupération idéologique, au cours du XIX ${ }^{e}$ siècle, par Charles Darwin qui lui confère un sens péjoratif : les peuples dont les avancées techniques sont jugées médiocres sont désignés comme primitifs, se situant au plus bas de l'échelle de l'évolution tandis que l'homme blanc, occidental, "civilisé ", domine le sommet de cette hiérarchie. Cette attitude condescendante envers le primitif, appréhendé comme arriéré ou attardé, est rattachée à l'histoire des conquêtes coloniales, qui se sont appuyées sur le classement des races. L'ethnologie moderne et contemporaine a révélé les apories d'un tel discours tout en réhabilitant le terme " primitif ", pour lequel l'anthropologue Mary Douglas précise :

Faut-il l'abandonner? Je ne le souhaite pas. En art, ce mot a un sens bien précis, et l'art primitif est entouré de respect. En technologie, et peut-être même en économie, le terme primitif pourrait trouver une acception valable. Ce qui caractérise la culture primitive, c'est $[\ldots]$ une vision personnelle, anthropocentrique et indifférenciée de l'univers. Où est le $\mathrm{mal}^{9}$ ?

Les caractéristiques des cultures primitives ne sont pas envisagées de manière négative et " primitif " n'équivaut plus à une déficience de qualités, mais devient un indice différentiel par rapport à la civilisation occidentale, témoignant aussi "d'un esprit d'invention et de réalisation qui laisse loin derrière lui les réussites des civilisés ${ }^{10}$ ". Dans le domaine de l'art, William Rubin assure que le terme a toujours été employé de manière laudative ${ }^{11}$. Au XIX ${ }^{\mathrm{e}}$ siècle, les arts qualifiés de "primitifs", admirés par les artistes pour leur simplicité et leur profondeur, sont multiples : on y regroupe les peintures de la pré-Renaissance, l'art grec ancien, l'artisanat paysan et les estampes japonaises. Au $\mathrm{Xx}^{\mathrm{e}}$ siècle, par le biais des cubistes, l'art primitif se spécifie pour qualifier principalement l'art tribal, venu d'Afrique et d'Océanie, à un moment où se noue un dialogue inédit entre artistes modernes et objets d'arts africains ou océaniens. Les codes de la représentation s'en trouvent fortement bouleversés, l'exigence mimétique est délaissée pour explorer les ressources formelles. Cette quête esthétique, éloignée de tout exotisme, se poursuit à

7. É. Souriau, Vocabulaire d'esthétique, PUF, $3^{e}$ édition, "Quadrige », 1999, p. 1239.

8. R. Goldwater, Le Primitivisme dans l'Art moderne, traduit de l'anglais par D. Paulme, PUF, 1998, p. 244. 9. M. Douglas, De la souillure. Essai sur les notions de pollution et de tabou, La Découverte, texte de l'édition de 1971, traduit de l'anglais par A. Guérin, 2012, p. 109.

10. C. Lévi-Strauss, Anthropologie structurale, [1958], Plon, 1971, p. 114.

11. W. Rubin, "Une introduction », dans W. Rubin (dir.), Le Primitivisme dans l'art du $20^{\circ}$ siècle, Flammarion, 1987, p. 11. 
travers l'engouement pour l'art des enfants et des aliénés. Fantasmatique, le primitif est avant tout l'autre, à la fois fascinant et inquiétant, ce qui conduit Henri Meschonnic à libérer le primitivisme du seul domaine de l'art tribal pour le définir comme « la figure la plus forte de l'altérité. De la tension entre identité et altérité ${ }^{12}$ ".

Dans un ouvrage récent, Valentine Plisnier a étudié le primitivisme photographique. L'appropriation d'œuvres issues de cultures extra-européennes par les photographes occidentaux constitue le fil rouge de son travail ${ }^{13}$. Bien que les photographies de Mengus et de Simon fassent écho au langage de l'art tribal, elles ne mettent pas en scène des sculptures d'Art primitif à la manière d'un Man Ray, immortalisant en 1926 un visage de femme blanche posant aux côtés d'un masque de femme noire. Dépassant le cadre théorique de Plisnier, nos deux photographes illustrent une forme de primitivisme qui s'intéresse principalement aux "primitifs de l'intérieur ${ }^{14}$ ". L' " autre " n'est plus situé dans des contrées lointaines, Afrique ou Océanie, mais se débusque jusque sur le sol européen.

\section{REPRÉSENTATION DU GITAN}

Dans Le Primitivisme et l'art moderne, Colin Rhodes souligne : "Géographiquement, les croyances européennes situaient le sauvage en Afrique centrale et australe, aux Amériques et en Océanie. Cependant, l'Occident lui-même avait depuis longtemps la conviction de posséder ses propres primitifs : les populations paysannes, les enfants et les fous ${ }^{15}$. " En effet, certaines cultures, bien qu'occidentales, sont perçues comme radicalement différentes, tout à la fois premières et immuables. Se tenant à l'écart des bouleversements occasionnés par la société technicienne, elles semblent donner une image de l’origine. Cherchant les primitifs à Perpignan, Mengus et Simon vont rencontrer la communauté gitane installée dans le quartier Saint-Jacques. Mengus est le premier à établir des relations avec les Gitans qu'il commence à photographier dès les années quarante. Lidentité du Tsigane contraste avec celle de l'homme social occidental, ce sédentaire attaché à la possession des biens

12. H. Meschonnic, op. cit., p. 272.

13. Voir V. Plisnier, Le Primitivisme dans la photographie : l'impact des arts extra-européens sur la modernité photographique de 1918 à nos jours, Trocadéro, 2012.

14. L'expression est de Colin Rhodes qui consacre un chapitre à ce motif dans son ouvrage, Le Primitivisme et l'art moderne, traduit de l'anglais par M. de Pracontal, Thames and Hudson, 1997.

15. Ibid., p. 7. 
fonciers et terriens. Quoiqu'installés dans un quartier de la ville catalane, les Gitans ne renoncent pas entièrement au nomadisme, puisqu'ils continuent à se déplacer saisonnièrement au gré des foires et des marchés.

Soit, tout d'abord, parmi les nombreuses photographies que Mengus a consacrées aux Gitans, cette scène à trois personnages ${ }^{16}$. Elle est remarquable tant l'art de composition apparaît manifeste. Les jeux d'ombre et de lumière confèrent à l'ensemble une dimension picturale. On pense aux tableaux du peintre espagnol Bartolomé Murillo, représentant de jeunes enfants en haillons dans des teintes de clair-obscur. Deux petites filles, habillées pauvrement, pieds nus, font face au jeune garçon vu de dos, dont la nudité cristallise le message primitiviste. Transparaît ici le mythe romantique qui alimente la littérature et les arts autour des gens du voyage. L'état naturel n'a pas disparu, le temps biblique des origines où l'homme n'avait pas à se vêtir et ne connaissait pas le péché est encore palpable chez certains peuples. Louer les sociétés moins développées sur le plan matériel appartient à une tradition de pensée qui remonte à l'Antiquité et qui prend un nouvel essor au XIX ${ }^{e}$ siècle. Selon la pensée rousseauiste, le Gitan deviendrait le bon sauvage; son mode de vie fruste est valorisé tandis que l'enfant est gage d'innocence et de pureté. Une anecdote narrée par Mengus témoigne de cette idéalisation de l'enfance :

Ma première apparition dans une assemblée gitane, dont l'aspect et le bruit, je l'avoue, m'intimidaient, ce fut sous la conduite - il me tenait par la main - d'un enfant de cinq ou six ans. Ses mines, son air protecteur étaient impayables. Et la gravité de l'instant ne m'empêchait pas de regretter de ne pouvoir mieux faire que les confier à mon souvenir ${ }^{17}$.

L’enfant devient l'intercesseur entre sociétés nomade et sédentaire. Étranger à la communauté qui semble, au premier abord, menaçante, le photographe est guidé par un être à la fois vulnérable et doté d'un grand pouvoir; " protecteur ", il possède les qualités des divinités tutélaires des religions archaïques. Ange gardien, il est celui par lequel l'étranger Mengus est accueilli. Peu étonnant que le photographe soit fasciné par le monde des enfants, portant une attention particulière à leurs jeux, comme le révèle cette photographie immortalisant un jeune garçon gitan faisant le poirier ${ }^{18}$. Un œil avisé reconnaitra l'enfant également photographié par Claude Simon aux côtés d'une rangée de camarades, complètement à droite, dans la photographie

16. Voir cahier iconographique, p. IV.

17. Les Carnets d'André Vick en Roussillon, "troisième cahier ", op. cit., p. 25.

18. Voir cahier iconographique, p. v. 
intitulée «Jeu " (Phot., p. $62^{19}$ ). Retrouver les mêmes visages au fil du travail photographique réalisé par les deux cousins révèle qu’ils ont bien fréquenté la même communauté et qu'ils partageaient une réelle connivence esthétique. Dans les deux clichés précédents, l'enfant est le reflet d'une vitalité et d'une liberté que ne parviennent pas à corseter les conventions de la société. Les cheveux sont ébouriffés, indisciplinés, les vêtements débraillés; l'enfant n’est pas seulement pur et bon comme nous l'enseignait le Romantisme, il est cet être dénué de savoir qui donne accès à une réalité que la culture réprime, il est cet autre, radicalement différent de l'adulte. L'infans, étymologiquement, celui qui ne parle pas, déploie un langage qui appartient au corps et ces photographies qui mettent en valeur le mouvement en témoignent précisément. Renverser l'ordre établi en mettant la tête à l'envers chez Mengus, former un groupe homogène qui semble batailler contre la fixité d'un mur chez Claude Simon. Les gestes, les sourires, les pleurs, tout ce qui touche au domaine affectif est privilégié. À la suite des grands conflits mondiaux, l'optimisme historique hérité des Lumières, le positivisme ou encore la foi dans la raison se sont vus contestés, étant jugés impropres à rendre compte du sens de l'Histoire ${ }^{20}$. À l'inverse, en se situant au début de l'évolution de toute vie humaine, l'enfance marque ce stade premier où dominent l'irrationnel et les sensations. Vierge de tout apprentissage, cet être en devenir est à même de porter un regard neuf sur le monde. Avec ces photographies d'enfants gitans, on accède à une sorte de primitivité au carré. Sont primitifs les Gitans, car désignés comme origine de l'humanité par le mythe romantique, tout autant que l'enfant, qui renvoie l'adulte à ses prémices.

Dans une autre photographie réalisée par Mengus ${ }^{21}$, un jeune garçon tient une bouteille à la main, saisi dans un moment de suspension, équilibriste entre ombre et lumière, ressuscitant l' "Homme-rat " de Simon (Phot., p. 43). Pourvu de traits singuliers, le garçon aux cheveux rasés et aux oreilles légèrement en pointe a séduit les deux photographes. L'attribut qu'est la bouteille vient renforcer l'étrangeté du personnage. Sa dimension fantastique est

19. Faute d'accord de l'ayant-droit, cette photographie, ainsi que les autres photographies réalisées par Claude Simon et mentionnées dans cet article n'ont pu être reproduites dans le cahier iconographique. Plusieurs d'entre elles sont néanmoins faciles à trouver en ligne. Voir avant-propos, p. 14 et 17 (note des éditeurs).

20. Dans La Route des Flandres, à travers l'évocation de la destruction de la bibliothèque de Leipzig, Claude Simon raille l'impuissance de la civilisation occidentale, ici symbolisée par l'objet livre, à empêcher les pires atrocités.

21. Voir cahier iconographique, p. vi. 
accentuée par Simon à l'aide du cadrage de près tandis que le titre souligne son animalité; être hybride, il est issu de l'imaginaire mythologique, et nous renvoie aux tréfonds, aux entrailles de la vie souterraine. Derrière le masque du civilisé, s'agite la bête primitive que cache tout individu.

Cette première série d'images, outre le fait qu'elle met en scène les mêmes individus, s'inscrit dans le mouvement photographique qui traverse l'aprèsguerre, représenté par des figures comme Doisneau, Boubat, Izis, Cartier-Bresson ou encore Dieuzaide. La photographie revient à la réalité, et se tourne vers l'homme des quartiers populaires. Elle se fait témoignage, saisissant les hommes dans le geste de leur métier. Les rues de Paris et les terrains vagues de banlieue sont un décor privilégié, le photographe y chemine et se laisse guider par le hasard des rencontres. La démarche de Mengus et de Simon n'est pas différente, bien qu'elle ait pour cadre principal la ville catalane à partir des années quarante. Marie de Thézy, qui a consacré un ouvrage à ce mouvement en révélant son "réalisme poétique ", remarque :

Quel que soit leur sujet, le regard des photographes humanistes est empreint de chaleur humaine. Les choses et les gens sont attachants et vrais [...]. Ces images expriment la volonté farouche de découvrir chez tous une étincelle de vie, de beauté, de bonté, de révéler la poésie cachée au cœur du réel le plus terne ${ }^{22}$.

La photographie humaniste se veut essentiellement réparatrice face à une société sous le choc des guerres mondiales. Il peut sembler déroutant que la démarche de Simon s'inscrive dans cette veine optimiste replaçant l'Homme au centre du monde. Au moment de la parution de l'album Photographies en 1992, l'écrivain-photographe accorde un entretien filmé où il nie toute influence directe de Cartier-Bresson ou de Doisneau et surtout, rejette en bloc «l'humanisme traditionnel ${ }^{23}$ ". Marqué par son expérience de cavalier durant la Seconde Guerre mondiale, il constate la faillite des valeurs occidentales, qui n'ont pu empêcher la barbarie la plus meurtrière. Dans ses romans, l'écrivain reste profondément pessimiste et aime rappeler que "l'homme [se trouve] parmi les choses ${ }^{24}$ ", renonçant à une vision anthropocentrique pour privilégier une conception plus proche de celles des peuples dits primitifs se percevant comme inclus dans une totalité. Dès lors, ses photographies

22. M. de Thézy, La Photographie humaniste : 1930-1960, histoire d'un mouvement en France, Contrejour, 1992, p. 17.

23. Émission Les Arts, « Claude Simon photographe », France 3 Régions, 16 mars 1992 (en ligne : [http:// www.ina.fr/video/I00013424], consulté le 15/09/2017). 
témoignent de son " rapport ambivalent à l'humanisme ${ }^{25}$ ", qui célèbre les hommes à travers le quotidien des Gitans, mais renonce à un réel ordonné, issu du rationalisme cartésien. Ainsi, formellement, certaines images simoniennes traduisent une volonté de renverser les catégories traditionnelles qui nous permettent de penser l'univers. De même, Mengus, cet homme du $\mathrm{XIX}^{\mathrm{e}}$ siècle, infléchit sa foi dans les valeurs idéalistes pour explorer une veine plus formaliste.

\section{DÉFORMATION DU RÉEL ET ANTROPOMORPHISME}

Représenter l'homme dans un univers rassurant contraste avec le projet primitiviste de la Modernité. En peinture, on abandonne la tradition de la mimesis, reposant sur la perspective linéaire et restituant un monde unifié dans un espace rationnel, où l'homme serait souverain. Dans les photographies de Mengus et de Simon, bien que l'on constate certaines caractéristiques propres à la photographie humaniste qui véhicule l'image d'un monde stable, certains éléments témoignent d'un changement de paradigme esthétique au sein duquel la suprématie des activités humaines est atténuée. La tradition réaliste qui en découle est aussi laissée de côté, Simon rappelant le rôle de transfiguration de l'art qui ne se contente pas de calquer le réel au sein d'un schéma d'ordre et de cohérence. Il précise : "Une œuvre impliquant la pensée, que ce soit un masque Dogon ou Esquimau, une cathédrale gothique, un concerto de Bach, une théorie de physique, une page de Proust ou une peinture de Paul Klee, est une tentative de conjuration, de prise de possession et de transformation de la nature et du monde par leur recréation dans un langage ${ }^{26}$."

En filigrane, l'écrivain critique l'école réaliste qui nourrit l'illusion de produire un simulacre du réel, minimisant la part d'artifice résidant dans toute création. La métamorphose à travers un langage est le noyau dur de toute démarche démiurgique. Il est peu surprenant que Claude Simon cite Paul Klee. En effet, l'artiste allemand fut l'un des premiers à s'intéresser à l'art des enfants et des fous, louant leur affranchissement des conventions mimétiques. Ce dernier insiste sur la nécessaire " déformation des réalités natu-

25. Voir C. Yapaudjian-Labat, «L'humanisme en question chez Claude Simon », dans P. Dirkx et P. Mougin (dir.), Claude Simon : Transitions, ENS éditions, 2011, p. 116 (en ligne : [https://books.openedition. org/enseditions/829?lang=fr], consulté le 03/04/2019).

26. C. Simon, «Littérature : tradition et révolution », La Quinzaine littéraire, 1-15 mai 1967, p. 13. 
relles à laquelle parvient l'artiste ${ }^{27} »$. Un passage extrait de sa Théorie de l'art moderne est particulièrement éclairant :

Tout d'abord, l'artiste n'accorde pas aux apparences de la nature la même importance contraignante que ses nombreux détracteurs réalistes. Il ne s'y sent pas tellement assujetti, les formes arrêtées ne représentant pas à ses yeux l'essence du processus créateur dans la nature. La nature naturante lui importe davantage que la nature naturée ${ }^{28}$.

Il ne faut pas se limiter à une univoque copie du réel mais « rendre visible l'invisible ${ }^{29}$ ", afin de donner une nouvelle vision de l'objet. Le projet de Simon et de Mengus n'est pas différent. La photographie, qui entretient des liens complexes avec la mimesis (puisque l'on peut faire l'expérience de la présence réelle de ce qui est photographié) ne renonce pas pour autant à transformer l'objet, à le dé-naturer pour révéler paradoxalement son essence véritable, sa véritable nature. Fin connaisseur de l'œuvre de Klee, Mengus a repris son langage géométrique si caractéristique. Comme dans les Arts Primitifs, des signes simples, non imitatifs, sont intégrés dans un jeu de formes.

Comme on peut le voir dans cette photographie d'une carafe ${ }^{30}$, la recherche d'une « inquiétante étrangeté », propre à l'univers surréaliste, permet de percevoir l'objet sous un jour nouveau. À travers cet assemblage incongru de cercle, de cylindre et de trapèze, une carafe dessine un corps humain dans l'esprit du tableau intitulé Senecio de Paul Klee, ou encore des hauts reliquaires Kota du Gabon. Fidèle à la démarche dadaïste, Mengus a recours au ready-made, obéissant à une poétique de la récupération et du détournement. L'absence de lumière élaborée trahit une quête d'immédiateté. Paradoxalement, il s'agit de découvrir un réel brut, insolite et sans artifice, qui puisse aussi transcender le monde familier.

Ce goût pour les formes architecturales minimalistes, saisissant la nature humaine dans une représentation qui ne doit plus rien aux canons de l'imitation ou de la figuration, est palpable dans une photographie d'un parterre composé de galets de rivière ${ }^{31}$. Mengus noue un dialogue avec l'art extra-occidental. Bien qu'il soit difficile de parler d'emprunts localisables, on constate que le photographe a assimilé et intégré le langage propre aux Arts Premiers, basé sur la simplification formelle et la disposition originale des éléments.

27. P. Klee, Théorie de l'art moderne, Genève, Gonthier, Bibliothèque Médiations, 1969, p. 28.

28. Ibid.

29. Ibid., p. 31.

30. Voir cahier iconographique, p. vII.

31. Voir cahier iconographique, p. vIII. 
Une autre photographie tourne définitivement le dos aux théories de l'imitation ${ }^{32}$ : la physionomie de cet objet-masque étonne par l'économie de moyens qu'elle déploie. Un récipient circulaire traçant un visage, deux trous indiquant la place des yeux et un repli faisant office de nez, suffisent à saisir la figure humaine dans une indéniable expressivité. Transformer le réel en formes simples permet de s'écarter de l'illusionnisme et de suivre la voie esthétique empruntée par les Arts extra-occidentaux.

Bien que Simon ait collectionné ces objets d'art, admirant les masques Baoulé dont le visage est en forme de disque ${ }^{33}$, ses photographies n'explorent pas, à la manière de son cousin, la géométrie évocatrice des objets à connotation anthropomorphe. Redéfinissant les contours de l'humanité, la célèbre série qu'il a consacrée aux platanes fait figure d'exception. " Jambes " et "Ventre et cuisses " (Phot., resp. p. 120 et 121) évoquent certaines sculptures monolithes archaïques et reflètent, par le choix du cadrage et leur titre respectif, les croyances des peuples primitifs. Comme l'a montré l'anthropologie, leurs schémas de pensée s'opposent au rationalisme occidental, qui sépare catégoriquement humains et non humains. À travers l'animisme qui dote la nature d'une âme, ces frontières sont abolies. Simon convoque cette façon ancestrale de percevoir le monde. Les branches deviennent des jambes tandis qu'un ventre et des cuisses apparaissent au sein de ce même tronc dont il a suffi d'inverser le sens de lecture. Ainsi, les motifs anthropomorphes confondent différents modes d'existence, ignorant les limitations de l'être humain. Philippe Descola a montré que pour les sociétés dites primitives, "les frontières de l'humanité ne s'arrêtent pas aux portes de l'espèce humaine ${ }^{34}$ ". Inversement, chez les Occidentaux, l'humain est extérieur et supérieur à la Nature, comme étranger à elle. L'anthropologue Mary Douglas précise le type de liens qu'entretiennent les peuples extra-occidentaux avec l'espace :

Les Aborigènes [...] considèrent la plupart des objets du ciel et de la terre comme des éléments d'un vaste système de signes [...]. L'aborigène ne se meut pas dans un paysage, mais dans un espace humanisé, saturé de significations ${ }^{35}$.

32. Voir cahier iconographique, p. Ix.

33. Remarque faite par L. Dällenbach dans le récit du voyage réalisé en compagnie de l'écrivain, voir L. Dällenbach, Claude Simon à New York, Genève, Zoé, 2013, p. 75.

34. Philippe Descola, "Avant-propos ", Par delà nature et culture, Gallimard, «Bibliothèque des sciences humaines ", 2009, p. 13.

35. M. Douglas, op. cit., p. 104. 
La forêt n'est pas un lieu sauvage à domestiquer ni un motif de délectation esthétique, mais elle est pourvue de sens et de symboles, elle est à déchiffrer. Le primitivisme simonien se nourrit de cette vision qui réconcilie l'humain avec son environnement proche. Dans le cadre d'une campagne d'abattage des platanes à Perpignan, Mengus a également consacré un ensemble de photographies à ces arbres majestueux. Intitulée "Mises à mort ", la série humanise le végétal, véhiculant une tonalité sombre et dramatique, en retraçant les étapes successives qui mènent à l'agonie finale où l'arbre est débité. Simon connaissait ce travail qui l'a sûrement influencé. Une vision très mélancolique et funèbre distingue Mengus, Simon préférant accentuer l'aspect brut et massif des arbres, perçus comme immarcescibles et fortement sexualisés.

Cependant, deux images issues de leurs séries respectives ne font pas de doute sur leur convergence esthétique. L'image de l'arbre à double tronc proposée par Mengus ${ }^{36}$ semble correspondre précisément à celui photographié par Simon, usant également du procédé d'inversion. Un détail en bas à droite marque une différence avec l'image simonienne : un morceau de toile, linge pansant peut-être une plaie ouverte sur le platane, semble marquer son déclin tout autant que sa mise à mort future. Mengus n'a pas donné de titre à sa photographie, mais il a noté au verso au crayon à papier : "Un platane du square : sa tête est dans le sol, il lève les jambes au ciel. " La même image métaphorique est convoquée, dévoilant un thème abondamment traité par les surréalistes. André Masson a réalisé de nombreux dessins opérant une synthèse entre entités végétales et humaines, où les corps s'entrelacent étroitement avec les branchages, à tel point qu'il est difficile de les distinguer. Avec les photographies de végétaux anthropomorphes, le primitivisme, qui apparaît ici, se nourrit des mythes ancestraux répertoriés par l'anthropologue Sir James George Frazer à la fin du XIX ${ }^{\mathrm{e}}$ siècle dans Le Rameau d'or. Considéré comme la Bible des surréalistes, l'ouvrage détaille de nombreux exemples de métamorphoses d'humains en végétaux au sein de cultures très différentes. Mengus et Simon ont recours à ce thème universel, qui traverse autant la culture gréco-latine que la culture tribale. À travers leur travail, on pense tout autant à l'épisode mythologique de Daphné se transformant en laurier qu'à certains rituels africains transformant la personne en une nouvelle entité spirituelle : ancêtre, animal ou végétal. 


\section{MATIÈRE BRUTE ET PRÉ-RATIONNELLE}

À partir des années 1950, la compréhension des peuples primitifs est renouvelée grâce aux travaux de Claude Lévi-Strauss donnant de nouveaux outils pour appréhender la mentalité primitive tout en critiquant les prétentions de la société technologique. Au même moment, l'art occidental porte une attention accrue au matériau, au concret, aux formes simples et, comme le remarque L. Dällenbach, on assiste à une " assomption de tout ce que la culture réprimait jusqu'alors comme ne relevant pas de la sphère esthétique ${ }^{37}$ ». Les substances pauvres, les résidus abîmés, patinés par le temps, sont dignes d'intérêt et renversent les canons traditionnels du Beau. Cette métamorphose du goût est à mettre en corrélation avec la découverte et l'appropriation des Arts primitifs par les artistes. Les ressemblances mimétiques ne suscitent plus autant d'intérêt tandis que des œuvres cultivant un "goût des différences ${ }^{38}$ " et créant des effets de surprise occupent le devant de la scène artistique.

En ne connaissant pas la tradition des matériaux nobles de l'Occident, les peuples primitifs explorent les ressources infinies de la matière à des fins rituelles et non pas seulement esthétiques. La photographie de Claude Simon intitulée "Page d'écriture " (Phot., p. 86 ${ }^{39}$ ) fait écho aux applications de boues et de galets agglutinés que l'on peut observer sur certains masques de la région amazonienne. Retrouver au sein des savoir-faire occidentaux les procédés matériels de l'art tribal signale une autre forme de primitivisme, attentif aux supports mal dégrossis et bruts. Bien que cette image de mur catalan trahisse une technique d'assemblage très organisée et méticuleuse, ce qui lui vaut d'ailleurs sa comparaison avec l'entreprise scripturale, ses composants, galets irréguliers et briques rongées par des lichens, nous indiquent un monde en cours de destruction, chaotique, imprévisible où la rigidité des concepts, leur mise en forme, leur saisie claire et distincte sont menacées. À travers cette photographie, on ne saurait trouver plus belle métaphore de la démarche d'écrivain de Claude Simon. Ces galets et briques hétérogènes figurent peutêtre ce que l'auteur nomme le «magma [...] de sensations ${ }^{40}$ " qui préexiste à l'écriture et où se joue une dialectique entre ordre et désordre.

37. L. Dällenbach, «La question primordiale », dans Sur Claude Simon, éd. cit., p. 67.

38. R. Schwab, "Renaissance de l'Archaïque ", La Gazette des Beaux-Arts, juillet-août, 1936, p. 1-20, cité dans D. Clévenot, "De la reconnaissance des arts primitifs à la transfiguration de l'archaïque ", Figures de l'art, "L'archaïque contemporain ", PUPPA, n 19, Pau, 2011, p. 61.

39. Voir [http://associationclaudesimon.org/claude-simon/iconographie/article/simon-claude-page-d-ecriture]. 40. C. Simon, Avant-propos, non paginé, Orion aveugle, Genève, Skira, 1970. 
Claude Simon admirait le travail de Jean Dubuffet avec qui il a entretenu une correspondance pendant une quinzaine d'années. Les " texturologies » et " matériologies " de l'artiste ont permis d'expérimenter des effets de matière valorisant l'indifférencié et l'innommable. La matière en cours de destruction s'exhibe et donne à voir ses composants les plus rebutants, éléments irréductibles qui précèdent la mise en forme élaborée. Les nombreux murs décrépis immortalisés par Simon reflètent une semblable recherche de l'informe et du pré-rationnel, renvoyant aux origines du monde. Quant à Mengus, il s'est intéressé abondamment aux surfaces vieillies. Son rejet du neuf, " morne et muet $^{41}$ ", s'accompagne d'une certaine réserve à l'égard des matières dégradées et viles. Voici ce qu'il note dans ses carnets :

On m’a reproché d'avoir trop de goût pour le vétuste, le croulant, alors que par exemple, les murs lépreux, je les déteste, qu'ils me rongent, que j'ai fait l'impossible pour les éliminer [...]. Il restait à prendre peu de choses, si l'on veut, un fond simple et humble, souvent presque nu, capable de mettre en valeur des détails ici et là : les pavés, les bordures des trottoirs, les pierres d'angles, le bois des contrevents et des boutiques, [...] le linge qui sèche, les visages qui se penchent, et, les mêmes gens allant à leurs affaires ou causant, parmi tous les enfants qui jouent $[\ldots]^{42}$.

La différence avec Simon est notable; sa préférence pour les éléments dotés d'une grande simplicité l'empêche d'apprécier les substances dégradées, aux contours incertains, multiples et complexes. Pour se faire une idée de sa façon d'appréhender la matière la plus brute, il suffit de regarder les photographies qu'il a consacrées à l'Art roman. D'abord réalisées dans un souci documentaire, ces images sont dotées d'une grande valeur esthétique. Ainsi, de cette photographie de bénitier en pierre ${ }^{43}$ que son caractère épuré entoure d'une aura mystérieuse, on remarque la récurrence des formes tant affectionnées par Mengus : le rectangle présent sur le mur, le trapèze soutenant le petit bassin, formant lui-même un demi-cercle. L'irrégularité du mur est atténuée par la géométrie des éléments, conférant une puissante stabilité à la matière, une assise inaltérable. À l'inverse, Simon privilégie les matières friables aux prises avec l'informe primitif. Cependant, un même goût rassemble à nouveau les deux cousins lorsqu'il s'agit d'appréhender les murs recouverts de graffitis de la ville catalane. Ces dessins non policés ${ }^{44}$, issus de la rue, témoigneraient

41. Les Carnets d'André Vick en Roussillon, " troisième cahier ", op. cit., p. 36.

42. Ibid., p. 37-38.

43. Voir cahier iconographique, p. XI.

44. Voir cahier iconographique, p. XII. Pour la photographie de graffiti de Claude Simon, il s'agit de "Portrait» (Phot., p. 49). 
d'une sauvagerie citadine qui est à rapprocher de l'expressivité de l'art tribal. En 1933, Brassaï a ouvert la voie lorsqu'il publie des photographies de murs parisiens dans la revue Minotaure. Selon l'artiste, " ces signes succincts ne sont rien moins que l'origine de l'écriture ${ }^{45}$ ». Revenir aux commencements obsède certains artistes de l'après-guerre qui souhaitent réaliser une tabula rasa pour repartir sur de nouvelles bases. Il n'est pas surprenant que l'on rapproche les graffitis des dessins pariétaux, comme en témoigne les analyses de l'écrivain suisse Charles-Albert Cingria :

De nos jours, il n’y a, à ce titre-là, que les graffiti des latrines qui méritent le nom de peinture. Cet art est désarçonnant. Je ne vois, dans le passé, qui puisse lui être comparé, en légèreté et en finesse d'une précision foudroyante [...] que celui-là bien français des grottes préhistoriques $[\ldots]^{46}$.

Le dessin trivial qui se développe en dehors de tout cadre normé, réalisé avec peu de moyens, supplante le plus haut degré de technicité promu par les écoles d'art, fleurons de culture et de savoir. Avec les graffitis, on accède à un art régressif qui ignore la virtuosité mimétique et qui, par un style heurté, hasardeux, dynamique, rappelle la liberté des gribouillis enfantins et des productions tribales. Afin d'accentuer le caractère prolifique de ces dessins, Mengus et Simon isolent le plus souvent un détail sur la surface du mur et, par une prise de vue rapprochée, transforment ces œuvres anonymes, leur conférant la force des productions laissées par des artistes comme Paul Klee, Jean Dubuffet ou Joan Miró.

Trois grands axes nous ont permis de comprendre le primitivisme déployé par nos deux photographes. Fidèles à la tradition romantique, ils désignent l'enfant Gitan comme un être originel, sauvage, non soumis aux lois du civilisé. Prenant leur distance avec la photographie humaniste et les normes de la mimesis réaliste, ils extirpent tout l'insolite de l'environnement familier en resserrant leur cadrage et en s'attachant aux détails, empruntant aussi la vision animiste des peuples primitifs. Enfin, ils questionnent la matière pré-rationnelle et informe, ou valorisent les principes géométriques élémentaires.

Dans l'introduction consacrée à l'album publié en 1992, Simon met l'accent sur la capacité de l'image photographique à garder une trace de ce qui ne sera plus jamais, insistant sur son pouvoir «magique» (Phot., p. 18). En employant ce terme, est-il possible que l'écrivain se rappelle les mots de

45. Brassaï, "Du mur des cavernes au mur d'usine ", Minotaure, no 3-4, déc. 1933.

46. C.-A. Cingria, "Art ancien - Art moderne ", Euvres Complètes, Lausanne, L'Âge d'homme, 19671978, tome 2, p. 333. 
Picasso définissant l'Art africain? "Raisonnable et magique ${ }^{47}$ ", nous dit le peintre, cherchant à révéler l'ambivalence de cet art, tout à la fois logique et irrationnel. Dans l'approche primitiviste qui est la nôtre, le rapport au temps semble le point commun entre la photographie et l'Art africain; cette convergence est suggérée par le terme "magique ", auquel deux artistes majeurs du $\mathrm{Xx}^{\mathrm{e}}$ siècle ont recours pour révéler la puissance évocatrice de deux formes artistiques, fracturant toutes deux les balises rationnelles et célébrant une temporalité singulière. En effet, l'Art africain entend présentifier le passé : la statuaire ne cherche pas à rendre hommage au révolu, mais réactualise le temps de l'objet représenté ${ }^{48}$. Pour Claude Simon, le dessein de la photographie n'est pas différent puisque ce médium ressuscite le passé, en figeant des moments présents. Une passerelle est lancée entre art primitif et art occidental et le primitivisme naît de cette rencontre. N'en doutons pas, la réflexion simonienne s'est nourrie de ce rapport au temps ancestral, qui court-circuite l'avancée linéaire et irréversible des époques ${ }^{49}$ et affirme que le passé ne disparaît jamais entièrement.

47. Propos rapportés par André Malraux dans La Tête d'obsidienne, Gallimard, 1974, p. 18, cité par William Rubin dans "Picasso ", Le Primitivisme dans l'art du $20^{\circ}$ siècle, éd. cit., p. 255.

48. Voir Jean Laude, La Peinture française et l'art nègre (1905-1914), Contribution à l'étude du fauvisme et du cubisme, [1968], Klincksieck, 2006, p. 337.

49. Pour une analyse plus détaillée du temps chez les peuples primitifs, voir l'ouvrage de Mircea Eliade, Le Mythe de l'éternel retour, Archétypes et répétition, [1949], Folio essais, 1995, p. 107. 